\title{
Facial Features Extraction by Relative Geometrical Position
}

\author{
Sundos A. Hameed \\ Al-Mustansiriyah University, \\ College of Sciences, \\ Computer Sciences Department
}

\begin{abstract}
In this research, traditional methods will be used to segment the true color of ace images such as Edge detection method and some preprocessing operations, the resulted regions was applied on the Facial Feature Extraction, for primary face image, segment the image into small and large regions (objects), the goal of this section is to detect the face region and facial features such as determining feature information about eyes, nose, and mouth, For facial feature extraction, one of very popular methods in geometrical feature based approach is the use of vertical axis of the center face region, so we used the face detection on facial database to recognized any input of gray face images, in this application the featurebased approach is selected. By testing many kind of images, some of which are face images, and all of these images prove the success of the applied it using proposed method.
\end{abstract}

\section{Keywords}

Segmentation, Facial Features, and Geometrical Position..

\section{INTRODUCTION}

The standard approaches to image analysis and recognition beings by segmentation of the image into regions and computing various properties and relationships among these regions. One of the most important tasks of an image analysis system is image segmentation, the identification of homogeneous regions in an image. The goal of the image segmentation is to divide an image into different regions that have homogeneous information [1,2], and to get the appropriate representation of the meaningful objects in the image with specific properties of interest. Segmentation is an extremely important operation in image analysis with several applications to pattern recognition, object detection, and medical image analysis [3], since it represents the very first step of low-level processing of imagery. For instance, in digital libraries large collections of images and videos need to be catalogued, ordered, and stored in order to efficiently browse and retrieve visual information [4].

Image segmentation can be applied in among numerous research fields, medical image: such as the detection of epileptic foci [5], X-ray images of the human chests where right and left lungs have to be segmented [6,7], and extract features of humans: such as Hand Detection, Face Detection, Facial Feature, and Face Recognition $[8,9,10]$. In this paper the region segmenting results of Edge Detection method are used and applied to some human face images. Many image segmentation techniques and more applications on it (such as: face detection and Facial feature) have been proposed during the past years, in some examples, (Srikathyayani S., M. Sc. Thesis, State University, Electrical and Computer Engineering, 1997), the basic idea of his research is that uses of full color imagery to locate defects will yield better segmentation results than can be obtained when only black and white imagery is used. (L. Tang, L. F. Tian, B.L. Steward, Information and Electrical Technologies, Div. of ASAE, 2000), they presented a supervised color image segmentation using a binary-coded genetic algorithm (GA) identifying a region in one of color space. (Wenmiao Lu and Shaohua Sun, Department of Electrical Engineering, Stanford University, 2003), an approach presented to automatic detection of human faces in color images, the proposed approach consists of three parts: a human skin segmentation to identify probable regions corresponding to human faces, and a view-based face detection to further identify the location of each human face..

\section{IMAGE SEGMENTATION}

The segmentation techniques are: Thresholding is computationally inexpensive and fast, it is the oldest segmentation method and is still widely used in simple applications. Detection of Discontinuities, a number of techniques for detecting points, lines, and edges are presented, the methods generally used to detect these properties are based on small spatial masks. Region Growing and Merging, region growing /merging is performed by finding homogeneous regions and changing them until they no longer meet the homogeneity criteria. Region growing techniques are generally better in noisy images, where borders are extremely difficult to detect. Clustering looks for data that can be grouped in domains other than the spatial domain.

\subsection{Edge Detection and Threshold}

The Sobel edge detection operator has been applied successfully to all three planes in the RGB space and the gradients were summed to obtain the resultant edges. We can compute the Sobel operator on each of the three RGB planes and then sum the results. For their map processing application where colors and objects are well defined, this seems to be an adequate technique for edge detection. However, for more complex color images where it is necessary to capture better the correlation between the planes, this approach would probably be inadequate $[13,14]$. The Sobel operators have the advantage of providing both a differencing and a smoothing effect. Because derivatives enhance noise, the smoothing effect is a particularly attractive feature of the Sobel operators.

Thresholding is one of the most important approaches to image segmentation. we can use the same basic approach and classify the point $(\mathrm{x}, \mathrm{y})$ as belonging to one object class if $\mathrm{T} 1$ $<\mathrm{f}(\mathrm{x}, \mathrm{y}) \leq \mathrm{T} 2$, to the other object class if $\mathrm{f}(\mathrm{x}, \mathrm{y})>\mathrm{T} 2$, and to the background if $\mathrm{f}(\mathrm{x}, \mathrm{y}) \leq \mathrm{T} 1$. This type of multi-level thresholding is less reliable than its single threshold counterpart because of the difficulty in establishing multiple thresholds that effectively isolate region of interest, especially when the number of corresponding histogram modes is large.

$\mathrm{T}=\mathrm{T}[\mathrm{x}, \mathrm{y}, \mathrm{p}(\mathrm{x}, \mathrm{y}), \mathrm{f}(\mathrm{x}, \mathrm{y})]$

where 
$\mathrm{f}(\mathrm{x}, \mathrm{y})$ : is the intensity of point $(\mathrm{x}, \mathrm{y})$ and $\mathrm{p}(\mathrm{x}, \mathrm{y})$ denotes some local property of this point.

\section{THE STRUCTURE OF THE SYSTEM}

The application of the results from our system is, the Face Detection and Facial Feature Extraction, for primary face image, segment the image into small and large regions (objects), the goal of this section is to detect the face region and facial features such as determining feature information about eyes, nose, and mouth.

For facial feature extraction, one of very popular methods in geometrical feature based approach is the use of vertical axis of the center face region.

The largest object among messed objects at certain range becomes candidate face region, and the image is converted to black and white using threshold method.

\subsection{Thınning an Image}

An important approach to represent the structural shape of a plane region is to reduce it, this reduction is the Skeleton of the region via a Thinning algorithm. Thinning procedure play a central role in a broad range of problems in image processing.From the last step in our system, those are binary regions $(\mathrm{B} / \mathrm{W})$. Region points which are assumed to have value 1 , and background points to have value 0 . This method consists of two basic steps applied to the contour point, P1 in Figure (1), is any block with value 1 and have at least one 8 neighbor value 0

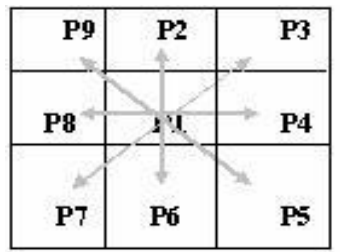

Figure (1) Neighbor arrangement used by the thinning algorithm

In our system, we need a thinning algorithm to reduce the points which are not important, this step produce a middle axis representation of a region.

\subsection{Median Filter}

The window (mask) size has been chosen as $3 \times 3$ because it is the smallest size that can be used to preserve the details of the image. It take with each pixel $\mathrm{P}$ the 8-connected neighbors $(\mathrm{P} 1, \mathrm{P} 2, \ldots, \mathrm{P} 9)$, and let the response of the filter at $\mathrm{P}$ is defined as the median of the pixel values at $\mathrm{P} 1, \mathrm{P} 2, \ldots, \mathrm{P} 9$, these values being ordered (increasing or decreasing matter) according to their intensity.

\subsection{Limitation}

The boundary of a region $\mathrm{R}$ is the set of pixel in the region that has at least one neighbor that are not in $\mathrm{R}$. if $\mathrm{R}$ happens to be an entire image (rectangular) rows and columns of the image. When one refers to a region, one refers to a subset of an image, and any pixels in the boundary of the region that happens to coincide with the border of the image are included implicitly as part of the region boundary.

From this binary image, we can easily calculate the area of object. The result will be a binary image with two regions, background and object. Obviously, calculating the area of object only needs scanning and getting the number of pixels in the object.
In this system, the location of any pixel (point) in the region is referred by $(\mathrm{X}, \mathrm{Y})$, all the pixels on the boundary of region have (X, Y).

In the Facial Feature Extraction part of this system, the traditional approach is followed using $\mathrm{B} / \mathrm{W}$ pixels and shape information. Preprocessing like thinning and median filter is required for this system in order to remove noise effect and to simplify the test image for Boy Image Figure (2).

Preprocessing steps include several traditional image processing methods which are applied together to obtain a better-input data for facial feature segmentation
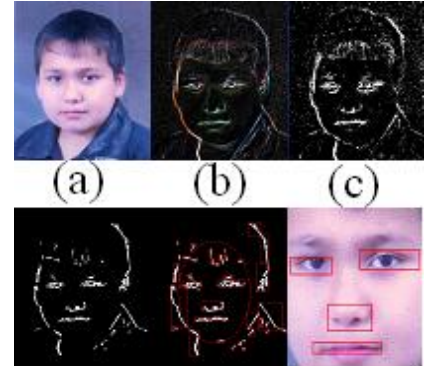

(d) (e)

(f)

Figure(2)Preprocessing operations: a-Origin Boy Image, b- Sobel Filter, c- B/W image, $d$ - Thinning and Median Filter, e- Limitation and d-Facial Feature Object

\section{FACIAL FEATURE EXTRACTION}

To detect the regions in the image for our application (Facial Feature Extraction) such as eyes, nose, mouth regions and the largest region (face), the feature is detected with enclosing rectangle. That means one should detect the effects regions, and then find for each region all the location pixels needed, such as Xmin, Xmax, Ymin, Ymax, Xcen, and Ycen.

From the face images, the face is the greatest part of the image, and hence the small object results from Image Segmentation which are connected with the largest object is the Face. All the objects circumscribe with the rectangle (Limitation).

First the ellipse mask is fitted on the largest object (face), the best - fit ellipse is computed on the basis of moments, and then the facial feature objects (eyes, nose, and mouth) are determined. Secondly, from the largest object the object is divided into three areas, and then we search from top to down in this region for a set of facial features which are grouped into face candidates. A vertical line is drawn from the upper point into lower point for the ellipse mask on the face region.

Step 1: Input Limited Regions

Step 2: From all the regions resulted, find the largest object.

Step 3: Determining all the regions (objects) that it's Center Location

(Xc, Yc) inside of the largest object.

Step 4: divide the object into three areas, by two horizontal lines.

Step 5: in the upper area, to detect pairs eyes objects:

A. examined if the object stand on the horizontal line 
B. there should be a symmetric pair, and the values of their distance are near, where the distance is the line between each center point and vertical line.

Step 6: in the middle area to detect the nose object:

A. it is located at lower than candidate eyes area, and it between them.

B. if there are several objects, select one, which has nearest to the point at vertical line.

Step 7: in the lower area, to detect the mouth object:

A. it located at lower than candidate nose position.

B. if there are several objects, select one with it horizontal distance is high and it center nearest to the vertical line

Step 8: at last the chin is located at lower than the mouth and at the point of ellipse and acts as the vertical line

.Step 9: Output, Eyes, nose, and mouth regions including with Face candidate region.

\section{EXPERIMENTAL AND RESULTS}

Ten male/female color images with different sizes and different backgrounds are used in this application of facial features Extraction.

After the segmentation stage, this application is completed with applying Preprocessing steps including several traditional image processing methods which are applied together to obtain a better input data for facial feature extraction application to the results regions, such as Thinning,Median Filter, and Limitation operations on the $\mathrm{B} / \mathrm{W}$ regions (Thresholding).

This section will display the results of the facial feature application steps, we most extract the features of face region (eyes, nose, and mouth), features will be extracted from the $\mathrm{B} / \mathrm{W}$ image, therefore the Sobel Operator is based on the Gray Scale Threshold $(0-255)$. The range of gray level is divided into five states, all the gray level values from our application are examined to sure if the Sobel method is good or not for the Facial Feature Application.

The ranges of the gray level values that are used in Sobel Operator are

State $1: 5-55$

State $2: 55-105$

State $3: 105-155$

State $4: 155-205$

State $5: 205-255$

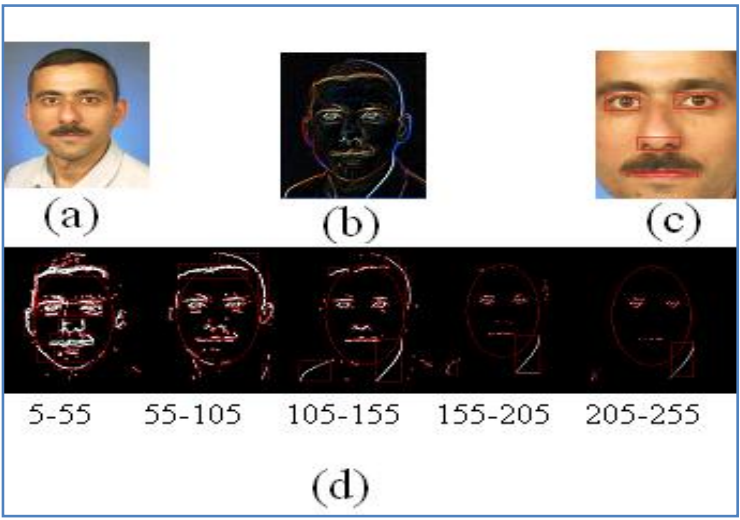

Figures (3) and (4) include the results of Limitation of regions, Face detection with ellipse mask, and the effect face region for our test images of human face (Man, and Woman)

Man: the width : 200 pixels , the length : 252 pixels, Threshold $=110$.

Figure (3) Results for Facial Feature extraction for MAN image: a- original image, b-Sobel Filter, c- effect Face region d- an image after thresholding with Limitation on the segmented regions.

Woman: the width : 320 pixels, the length : 355 pixels, Threshold $=70$

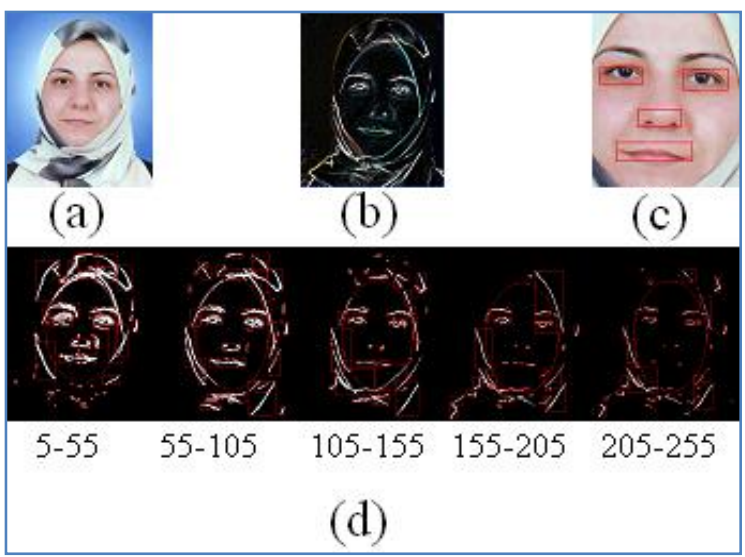

Figure (4) Results for Facial Feature extraction for WOMAN image: a- original image, b-Sobel Filter, c- effect Face region d- an image after thresholding with Limitation on the segmented regions

Human face may change its appearance because of facial expression, beard, moustache, hairstyle, make-up, glasses, aging, surgery, etc. In addition to these internal variations, external distortions such as the scale, lighting, and position of face must also be considered.

In this stage, geometrical features and relation value of features may show some differences with people, so it is suggested five values (states) to determine the Threshold value.

All the test images will be applied to its threshold, but if the results of Facial Feature are not extracted on this value this threshold value must be changed to other value by chosen a different state ( from five parts threshold(states)). The human face images along with the segmented and extraction of its features are shown in Tables (1) for Boy test Image, Table (2) for Man test image, and Table (3) for Woman Test Image, so 
the different values of the Threshold needed to decided if the Facial Feature application is successful in this threshold or failure.

Table(1) Boy Test image Values

\begin{tabular}{|c|c|}
\hline Threshold Value & Result of test \\
\hline 40 & Failure \\
\hline 74 & Access \\
\hline 122 & Access \\
\hline 190 & Poor \\
\hline 210 & Failure \\
\hline
\end{tabular}

Table(2) Man Test image Values

\begin{tabular}{|c|c|}
\hline Threshold Value & Result of test \\
\hline 40 & Failure \\
\hline 70 & Access \\
\hline 130 & Poor \\
\hline 200 & Poor \\
\hline 221 & Failure \\
\hline
\end{tabular}

Table(3) Woman Test image Values

\begin{tabular}{|c|c|}
\hline Threshold Value & Result of test \\
\hline 42 & Failure \\
\hline 75 & Poor \\
\hline 130 & Access \\
\hline 189 & Poor \\
\hline 211 & Failure \\
\hline
\end{tabular}

where:

Access : All the features of face image are extracted successfully (pair eyes, nose, and mouth).

Poor : The feature of face image is not extracted successfully (at least one of the facial feature is not extracted, eyes, nose, or mouth) .

Failure : The feature of face image is not extracted, or the system does not responded to this value

\section{CONCLUSION AND FUTURE WORK}

In this paper, image segmentation is work with edge detection and threshold methods, and facial feature extraction is also discussed (eyes, nose, or mouth).

From this segmentation method and facial feature application the following conclusions are drawn:

In limitation step, when one refers to a region, one refers to a subset of an image, and any pixels in the boundary of the region that happens to coincide with the border of the image are included implicitly as part of the region boundary.

With some example images along with the segmented feature, the result of facial feature detection application is poor because of existence of an expression in the face, or the rotation of the face.

If the face orientation is front view, the results are better, if there is a rotation on the face, some part is not detected.

For future work using this system:

On the resulting regions, one can examine another application, such as medical images.
From this application, the result of face detection and its feature can be used to design and improve the face recognition system.

\section{ACKNOWLEDGMENTS}

My thanks to all staff at my Computer Sciences Department in Al-Mustansiriyah University, College of Sciences.

\section{REFERENCES}

[1] S.E. Umbaugh, "Computer Vision and Image Processing”, Prentice-Hall, 1998.

[2] R. Muller, "Introduction into Image Analysis", Computer Graphics and Image Processing, GDV.RM.WS02/ 0310, 2002, www.cs.rotgers.edu.

[3] Karaman E., Hassanpour R., "Model Based Human Face Segmentation Using Skin Color", Cankaya University, Turkish Symposium on Artificial Intelligence and Neural Network, 2003.

[4] Aladdin J. Al-Hilli, "A Fuzzy Clustering Approach For Colored Image Segmentation”, Ph.D. thesis ,Computer Sciences, Higher Studies Institute for Computers \& Information, 2002

[5] Baogang W., Dongming L., Yunhe P., and Wenhua X., "Interactive Image Segmentation Using Multiple Color Spaces And Its Application in Ancient Art Preservation", Artificial Intelligence Institute, Zhejiang University, Hangzhou, 2000, China P. R. 310027, www.rostock.zgdv.de.

[6] Silver B., " An Introduction to Digital Image Processing", Cognex Corporation, One Vision Drive, Natick, MA 01760, 2000.

[7] Gonzalez R. C. and Woods E. , "Digital image processing", Addison-Wesley Publishing Company, 2004.

[8] Shant K.A., "Colored Image Segmentation Using Fuzzy Sets", University of Technology, M. Sc. Thesis, 1997.

[9] Tang L., Tian L. F., and Steward B.L., "Color Image Segmentation with Genetic Algorithm for In-Field Weed Sensing “, Information and Electrical Technologies, Div. of ASAE, 2000.

[10] Sural S., Qian G. and Pramanik S., "Segmentation and Histogram Generation using the HSV Color Space for Image Retrieval", IEEE, ICIP, pp II 589 - II 592, 2002.

[11] Baskan S., Bulut M. M., and Atalay V., "Projection based method for Segmentation of Human Face and its evaluation", Elsevier Scince, pp 1623-1629, 2002,www.elsevir.com.

[12] Lu W., and Sun S., "Face Detection in Color Images ", Department of Electrical Engineering, Stanford University, 2003

[13] Wesolkowski S. B., "Color Image Edge Detection and Segmentation: A Comparison of the Vector Angle and the Euclidean Distance Color Similarity Measures “, M. Sc. Thesis, University of Waterloo, 1999.

[14] Wesolkowski S., and Jernigan E., "Color Edge Detection in RGB Using Jointly Euclidean Distance And Vector Angle ", University of Waterloo, canada, pp.9-16, 1999. 